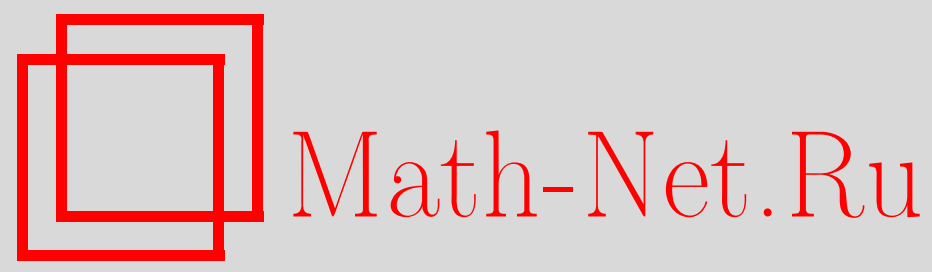

А. А. Кельзон, Определение скачка функции обобщенной ограниченной вариации с помощью производных от тригонометрического интерполяционного полинома, Матем. заметки, 2004, том 76, выпуск 1, 78-86

DOI: https://doi.org/10.4213/mzm90

Использование Общероссийского математического портала Math-Net.Ru подразумевает, что вы прочитали и согласны с пользовательским соглашением http://www.mathnet.ru/rus/agreement

Параметры загрузки:

IP: 52.23 .180 .231

26 апреля 2023 г., $17: 17: 24$

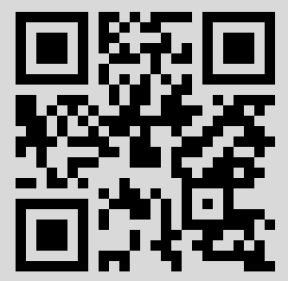




\section{ОПРЕДЕЛЕНИЕ СКАЧКА ФУНКЦИИ ОБОБЩЕННОЙ ОГРАНИЧЕННОЙ ВАРИАЦИИ С ПОМОЩЬЮ ПРОИЗВОДНЫХ ОТ ТРИГОНОМЕТРИЧЕСКОГО ИНТЕРПОЛЯЦИОННОГО ПОЛИНОМА}

\section{А. А. Кельзон}

Получены формулы, выражающие величину скачка ограниченной периодической функции, имеющей гармоническую ограниченную вариацию в окрестности рассматриваемой точки, через производные нечетного порядка от тригонометрического интерполяционного полинома Лагранжа по равноотстоящим узлам и через производные четного порядка от сопряженного ему полинома.

Библиографоия: 14 названий.

Пусть $f-2 \pi$-периодическая функция; $S_{n}(f ; x)$ и $\widetilde{S}_{n}(f ; x)$ - частные суммы порядка $n$ тригонометрического ряда Фурье и ряда, сопряженного к ряду Фурье этой функции, соответственно. Б. И. Голубов [1] доказал, что если $f$ имеет ограниченную $p$-вариацию $(p \geqslant 1)$ в смысле Н. Винера [2], то во всякой точке $x^{\prime}$ справедливы равенства

$$
\begin{array}{cl}
\lim _{n \rightarrow \infty} \frac{S_{n}^{(2 r+1)}\left(f ; x^{\prime}\right)}{n^{2 r+1}} & =\frac{(-1)^{r}}{(2 r+1) \pi}\left(f\left(x^{\prime}+\right)-f\left(x^{\prime}-\right)\right), \quad r=0,1,2, \ldots, \\
\lim _{n \rightarrow \infty} \frac{\widetilde{S}_{n}^{(2 r)}\left(f ; x^{\prime}\right)}{n^{2 r}} & =\frac{(-1)^{r+1}}{2 r \pi}\left(f\left(x^{\prime}+\right)-f\left(x^{\prime}-\right)\right), \quad r=1,2, \ldots
\end{array}
$$

Отметим, что частный случай формулы (1) при $r=0$ для более узких классов функций был известен ранее [3], [4] (см. также [5, гл. III, § 9]).

В работах [6], [7] справедливость формул (1) и (2) была установлена для более широкого класса функций, имеющих на периоде гармоническую ограниченную вариацию [8] (определение см. ниже).

Возникает вопрос, нельзя ли для определения скачка периодической функции вместо производных от сумм Фурье использовать производные от тригонометрических интерполяционных полиномов Лагранжа по равноотстоящим узлам, для вычисления которых, как известно, достаточно знать значения функции лишш на счетном множестве точек периода. Для функций ограниченной $p$-вариации положительный ответ на этот вопрос был получен в [9]. В настоящей заметке этот результат обобщается на функции, имеющие гармоническую ограниченную вариацию в некоторой окрестности рассматриваемой точки. 
Основные обозначения и формулировки результатов. Пусть $\left\{I_{n}\right\}, n \in \mathbb{N}$, последовательность непересекающихся интервалов $I_{n}=\left(a_{n}, b_{n}\right) \subset[a, b]$. Для функции $f$, заданной на отрезке $[a, b]$, положим $f\left(I_{n}\right)=f\left(b_{n}\right)-f\left(a_{n}\right)$ и пусть

$$
V_{H}(f ; a, b):=\sup \sum_{n=1}^{\infty} \frac{\left|f\left(I_{n}\right)\right|}{n}
$$

где верхняя грань берется по всевозможным последовательностям интервалов $\left\{I_{n}\right\}$, удовлетворяющим описанным условиям. Если $V_{H}(f ; a, b)<\infty$, то говорят, что $f$ имеет гармоническую ограниченную вариацию на $[a, b]$. Класс таких функций обозначим через $\mathrm{HBV}[a, b]$.

Пусть $\xi$-произвольное вещественное число. Для $2 \pi$-периодической функции $f$ через $L_{n, \xi}(f ; x), n=0,1 \ldots$, обозначим тригонометрический полином порядка не вьше $n$, совпадающий с функцией $f$ в узлах

$$
x_{j, n} \equiv x_{j, n}(\xi)=\xi+2 j \frac{\pi}{2 n+1}, \quad j=0, \pm 1, \ldots
$$

(тригонометрический интерполящионньй полином Лагранжа).

Как известно (см., например, [10, с. 509]),

$$
L_{n, \xi}(f ; x)=\frac{2}{2 n+1} \sum_{j=-n}^{n} f\left(x_{j, n}\right)\left(\frac{1}{2}+\sum_{k=1}^{n} \cos k\left(x-x_{j, n}\right)\right)
$$

или

$$
L_{n, \xi}(f ; x)=\frac{a_{0}^{(n)}(f)}{2}+\sum_{k=1}^{n}\left(a_{k}^{(n)}(f) \cos k x+b_{k}^{(n)}(f) \sin k x\right) \text {, }
$$

где

$$
\begin{aligned}
& a_{k}^{(n)}(f)=\frac{2}{2 n+1} \sum_{j=0}^{2 n} f\left(x_{j, n}\right) \cos k x_{j, n}, \quad k=0, \ldots, n, \\
& b_{k}^{(n)}(f)=\frac{2}{2 n+1} \sum_{j=0}^{2 n} f\left(x_{j, n}\right) \sin k x_{j, n}, \quad k=1, \ldots, n,
\end{aligned}
$$

- коэффициенты Фурье-Лагранжа функции $f$.

Пусть далее $\tilde{L}_{n, \xi}(f ; x), n=1,2, \ldots$, - тригонометрический полином, сопряженньй к $L_{n, \xi}(f ; x)$, т.е.

$$
\tilde{L}_{n, \xi}(f ; x)=\sum_{k=1}^{n}\left(a_{k}^{(n)}(f) \sin k x-b_{k}^{(n)}(f) \cos k x\right) .
$$

Положим, наконец,

$$
J_{m}:=\left(\frac{2}{\pi}\right)^{m} \int_{0}^{\pi / 2} \frac{x^{m}}{\sin x} d x, \quad m \in \mathbb{N}
$$


ТЕОРема 1. Пусть $f-2 \pi$-периодическая ограниченная функиия $u r=0,1, \ldots$. Тогда

а) если на некотором отрезке $[a, b]$, содержащем внутри себя точку $x^{\prime}, f$ имеет гармоническую ограниченную вариачию, то справедливо равенство

$$
\lim _{n \rightarrow \infty} \frac{L_{n, x^{\prime}-\pi}^{(2 r+1)}\left(f ; x^{\prime}\right)}{n^{2 r+1}}=\frac{(-1)^{r}}{\pi} J_{2 r+1}\left[f\left(x^{\prime}+\right)-f\left(x^{\prime}-\right)\right]
$$

б) не существует способа для определения скачка в точке $x^{\prime}$ произвольной $2 \pi$-периодической функиии $f \in \mathrm{HBV}[0,2 \pi]$ через последовательность $\left\{L_{n, x^{\prime}-\pi}^{(2 r)}\left(f ; x^{\prime}\right)\right\}, n=0,1, \ldots$.

Теорема 2. Пусть $f-2 \pi$-периодическая ограниченная функиия $u r=1,2, \ldots$. Tогда

а) если на некотором отрезке $[a, b]$, содержащем внутри себя точку $x^{\prime}, f$ имеет гармоническую ограниченную вариачию, то справедливо равенство

$$
\lim _{n \rightarrow \infty} \frac{\tilde{L}_{n, x^{\prime}-\pi}^{(2 r)}\left(f ; x^{\prime}\right)}{n^{2 r}}=\frac{(-1)^{r+1}}{\pi} J_{2 r}\left[f\left(x^{\prime}+\right)-f\left(x^{\prime}-\right)\right]
$$

б) не существует способа для определения скачка в точке $x^{\prime}$ произвольной $2 \pi$-периодической функиии $f \in \operatorname{HBV}[0,2 \pi]$ через последовательность $\left\{\tilde{L}_{n, x^{\prime}-\pi}^{(2 r-1)}\left(f ; x^{\prime}\right)\right\}, \quad n=1,2, \ldots$.

Доказательства. Мы ограничимся доказательством теоремы 1, ибо теорема 2 доказывается аналогично.

Заметим, что, не уменьшая общности, мы можем считать $x^{\prime}=\pi$ (иначе можно сделать линейную замену переменной). Теперь у нас $x_{j, n}=2 j \pi /(2 n+1)$. Далее вместо $L_{n, 0}(f ; x)$ будем писать $L_{n}(f ; x)$.

Рассмотрим функцию $f_{0}$, определяемую равенствами $f_{0}(x)=x$ при $-\pi<x \leqslant \pi$, $f_{0}(x+2 \pi)=f_{0}(x) \forall x \in \mathbb{R}$, и докажем вначале справедливость формулы (5) для $f=f_{0}$. Имеем из (4)

$$
L_{n}\left(f_{0} ; x\right)=\frac{2}{2 n+1} \sum_{j=-n}^{n} x_{j, n}\left(\frac{1}{2}+\sum_{k=1}^{n} \cos k\left(x-x_{j, n}\right)\right) .
$$

Отсюда

$$
L_{n}^{(2 r+1)}\left(f_{0} ; \pi\right)=\frac{2(-1)^{r+1}}{2 n+1} \sum_{k=1}^{n}(-1)^{k+1} k^{2 r+1} \sum_{j=-n}^{n} \frac{2 j \pi}{2 n+1} \sin j \frac{2 k \pi}{2 n+1} .
$$

Ho $[11$, c. 45$]$

$$
\sum_{j=-n}^{n} \frac{2 j \pi}{2 n+1} \sin \left(j \frac{2 k \pi}{2 n+1}\right)=\frac{4 \pi}{2 n+1} \sum_{j=1}^{n} j \sin \left(j \frac{2 k \pi}{2 n+1}\right)=\frac{(-1)^{k+1} \pi}{\sin \frac{k \pi}{2 n+1}}
$$


Следовательно,

$$
L_{n}^{(2 r+1)}\left(f_{0} ; \pi\right)=(-1)^{r+1} \frac{2 \pi}{2 n+1} \sum_{k=1}^{n} \frac{k^{2 r+1}}{\sin \left(\frac{k \pi}{2 n+1}\right)}
$$

откуда

$$
\begin{aligned}
\lim _{n \rightarrow \infty} \frac{L_{n}^{(2 r+1)}\left(f_{0} ; \pi\right)}{n^{2 r+1}} & =2(-1)^{r+1}\left(\frac{2}{\pi}\right)^{2 r+1} \lim _{n \rightarrow \infty} \frac{\pi}{2 n+1} \sum_{k=1}^{n} \frac{\left(\frac{k \pi}{2 n+1}\right)^{2 r+1}}{\sin \frac{k \pi}{2 n+1}} \\
& =\frac{(-1)^{r}}{\pi} J_{2 r+1}\left(f_{0}(\pi+)-f_{0}(\pi-)\right),
\end{aligned}
$$

т.е. (5) доказано для $f=f_{0}$ и $x^{\prime}=\pi$.

Пусть теперь дана произвольная ограниченная $2 \pi$-периодическая функция $f \in$ $\operatorname{HBV}[a, b], \pi \in(a, b)$ и $d=f(\pi+)-f(\pi-)$. Положим

$$
F(x):=f(x)+\frac{d}{2 \pi} f_{0}(x)
$$

Очевидно, $F(\pi+)=F(\pi-)$ и $F \in \operatorname{HBV}[a, b]$. В силу линейности интерполяционного оператора ясно также, что, если мы докажем справедливость формулы (5) для функции $F$, то она будет справедлива и для $f$.

Итак, дана $2 \pi$-периодическая ограниченная функция $F \in \operatorname{HBV}[a, b]$, непрерьвная в точке $\pi \in(a, b)$. Не уменьшая общности, будем считать, что $F(\pi)=0$. Для доказательства утверждения а) теоремы 1 осталось показать, что

$$
\lim _{n \rightarrow \infty} \frac{L_{n}^{(2 r+1)}(F ; \pi)}{n^{2 r+1}}=0, \quad r=0,1, \ldots
$$

Пусть $D_{n}(x)$ - ядро Дирихле, т.е.

$$
D_{n}(x)=\frac{\sin (n+1 / 2) x}{2 \sin (x / 2)}=\frac{1}{2}+\sum_{k=1}^{n} \cos k x, \quad n \in \mathbb{N}
$$

В этих обозначениях

$$
L_{n}(F ; x)=\frac{2}{2 n+1} \sum_{j=0}^{2 n} F\left(x_{j, n}\right) D_{n}\left(x-x_{j, n}\right),
$$

откуда

$$
L_{n}^{(2 r+1)}(F ; \pi)=\frac{1}{2 n+1} \sum_{j=0}^{2 n} F\left(x_{j, n}\right) d_{j},
$$

где для краткости положено

$$
d_{j} \equiv d_{j, n, r}:=2 D_{n}^{(2 r+1)}\left(\pi-x_{j, n}\right)
$$


Зададим произвольное $\varepsilon>0$. Так как $F \in \operatorname{HBV}[a, b]$ и непрерьвна в точке $\pi \in(a, b)$, то [12] можно подобрать $\delta \in(0, \pi)$ такое, что $V_{H}(F ; \pi-\delta, \pi+\delta)<\varepsilon$. Пусть натуральное $s=s(n)$ выбрано из условия $x_{n-s, n} \leqslant \pi-\delta<x_{n-s+1, n}$.

Продифференцировав $2 r+1$ раз правую часть $(7)$, найдем, что $d_{0}=2 D_{n}^{(2 r+1)}(\pi)=0$.

Введем обозначения:

$$
\begin{aligned}
& A_{n} \equiv A_{n, r}:=\frac{1}{2 n+1} \sum_{j=1}^{n-s} F\left(x_{j, n}\right) d_{j}, \\
& B_{n} \equiv B_{n, r}:=\frac{1}{2 n+1} \sum_{j=n-s+1}^{n} F\left(x_{j, n}\right) d_{j}, \\
& E_{n} \equiv E_{n, r}:=\frac{1}{2 n+1} \sum_{j=n+1}^{n+s} F\left(x_{j, n}\right) d_{j}, \\
& G_{n} \equiv G_{n, r}:=\frac{1}{2 n+1} \sum_{j=n+s+1}^{2 n} F\left(x_{j, n}\right) d_{j} .
\end{aligned}
$$

Перепишем теперь (8) в виде

$$
L_{n}^{(2 r+1)}(F ; \pi)=A_{n}+B_{n}+E_{n}+G_{n} .
$$

Продифференцировав $2 r+1$ раз равенство (7), получим

$$
2 D_{n}^{(2 r+1)}(x)=\sum_{l=0}^{2 r+1} C_{2 r+1}^{l}\left(\frac{1}{\sin (x / 2)}\right)^{(l)}\left(n+\frac{1}{2}\right)^{2 r+1-l} \sin \left(\left(n+\frac{1}{2}\right) x+(2 r+1-l) \frac{\pi}{2}\right) .
$$

Легко видеть, что

$$
\left|\left(\frac{1}{\sin (x / 2)}\right)^{(l)}\right| \leqslant \frac{C(l)}{\left|\sin ^{l+1}(x / 2)\right|}, \quad l=0,1, \ldots
$$

где $C(l)$ зависит только от $l$.

Поскольку

$$
\sin \left(\left(n+\frac{1}{2}\right)\left(\pi-x_{j, n}\right)+(2 r+1-l) \frac{\pi}{2}\right)=\sin \left(\left(n+r+1-j-\frac{l}{2}\right) \pi\right)
$$

что равно нулю при четных $l$, то при $x=\pi-x_{j, n}$ суммирование в правой части (10) фактически проводится только по нечетным $l$ и, в частности, начинается $c l=1$. Далее при $j=1, \ldots, n-s \sin \left(\left(\pi-x_{j, n}\right) / 2\right) \geqslant \sin (\delta / 2)$ и из (10) и (11) следует, что при указанных $j d_{j}=O\left(n^{2 r}\right), n \rightarrow \infty$. Отсюда в силу ограниченности $F$

$$
A_{n}=\frac{1}{2 n+1} \sum_{j=1}^{n-s} F\left(x_{j, n}\right) d_{j}=O\left(n^{2 r}\right), \quad n \rightarrow \infty
$$


Аналогично показывается, что

$$
G_{n}=O\left(n^{2 r}\right), \quad n \rightarrow \infty
$$

Переходим к оценке $B_{n}$. Имеем (см. формулу (38) в [7])

$$
D_{n}(t)=\frac{\sin n t}{t}+\xi(t) \sin n t+\frac{1}{2} \cos n t
$$

где $\xi(t)=(1 / 2) \operatorname{ctg}(t / 2)-1 / t$ при $0<|t|<2 \pi$ и $\xi(0)=0$.

Продифференцировав равенство (14) $2 r+1$ раз, получим

$$
\begin{aligned}
d_{j}= & 2 D_{n}^{(2 r+1)}\left(\pi-x_{j, n}\right)=2 n^{2 r+1} \frac{\sin \left((n+r+1 / 2) \pi-n x_{j, n}\right)}{\pi-x_{j, n}} \\
& +2 \sum_{i=0}^{2 r} C_{2 r+1}^{i} n^{i} \sin \left(n \pi-n x_{j, n}+\frac{i \pi}{2}\right)(-1)^{1-i}(2 r+1-i) !\left(\pi-x_{j, n}\right)^{i-2 r-2} \\
& +2 \sum_{i=0}^{2 r+1} C_{2 r+1}^{i} \xi^{(2 r+1-i)}\left(\pi-x_{j, n}\right) n^{i} \sin \left(\left(n+\frac{i}{2}\right) \pi-n x_{j, n}\right) \\
& +n^{2 r+1} \cos \left(\left(n+r+\frac{1}{2}\right) \pi-n x_{j, n}\right) .
\end{aligned}
$$

Известно (см., например, [13, с. 484]), что $\xi(t)$ - аналитическая функция в интервале $(-2 \pi, 2 \pi)$ и, следовательно, имеет конечные производные всех порядков. Поэтому

$$
\begin{aligned}
d_{j}= & 2 n^{2 r+1}(-1)^{n+r} \frac{\cos n x_{j, n}}{\pi-x_{j, n}} \\
& +2 \sum_{i=0}^{2 r} C_{2 r+1}^{i} n^{i}(-1)^{n+1-i} \sin \left(\frac{i \pi}{2}-n x_{j, n}\right)(2 r+1-i) !\left(\pi-x_{j, n}\right)^{i-2 r-2} \\
& +2 \xi\left(\pi-x_{j, n}\right) n^{2 r+1}(-1)^{n+r} \cos n x_{j, n} \\
& +n^{2 r+1}(-1)^{n+r} \sin n x_{j, n}+O\left(n^{2 r}\right), \quad n \rightarrow \infty
\end{aligned}
$$

Заметив, что

$$
\begin{aligned}
\xi\left(\pi-x_{j, n}\right) & =\frac{1}{2} \operatorname{tg} \frac{x_{j, n}}{2}-\frac{1}{\pi-x_{j, n}} \\
\cos n x_{j, n} & =(-1)^{j} \cos \left(x_{j, n} / 2\right) \\
\sin n x_{j, n} & =(-1)^{j+1} \sin \left(x_{j, n} / 2\right)
\end{aligned}
$$

из (15) получим

$$
d_{j}=2 \sum_{i=0}^{2 r} C_{2 r+1}^{i} n^{i}(-1)^{n+1-i} \sin \left(\frac{i \pi}{2}-n x_{j, n}\right)(2 r+1-i) !\left(\pi-x_{j, n}\right)^{i-2 r-2}+O\left(n^{2 r}\right)
$$


Далее имеем, учитывая ограниченность $F$,

$\sum_{j=n-s+1}^{n} F\left(x_{j, n}\right) d_{j}=2 \sum_{i=0}^{2 r}(-1)^{n+1-i} C_{2 r+1}^{i} n^{i}(2 r+1-i) ! \sum_{j=n-s+1}^{n} F\left(x_{j, n}\right) l_{i, j}+O\left(n^{2 r+1}\right)$,

где

$$
l_{i, j} \equiv l_{i, j, n}=\frac{\sin \left(i \pi / 2-n x_{j, n}\right)}{\left(\pi-x_{j, n}\right)^{2 r+2-i}} .
$$

Будем изучать поведение внутренней суммы в правой части (19). Имеем, с учетом (16) и (17)

$$
l_{i, j}= \begin{cases}\frac{(-1)^{j} \sin \left(x_{j, n} / 2\right)}{\left(\pi-x_{j, n}\right)^{2 r+2-i}}, & i \equiv 0(\bmod 4) \\ \frac{(-1)^{j} \cos \left(x_{j, n} / 2\right)}{\left(\pi-x_{j, n}\right)^{2 r+2-i}}, & i \equiv 1(\bmod 4) \\ \frac{(-1)^{j+1} \sin \left(x_{j, n} / 2\right)}{\left(\pi-x_{j, n}\right)^{2 r+2-i}}, & i \equiv 2(\bmod 4) \\ \frac{(-1)^{j+1} \cos \left(x_{j, n} / 2\right)}{\left(\pi-x_{j, n}\right)^{2 r+2-i}}, & i \equiv 3(\bmod 4)\end{cases}
$$

Очевидно, что функция

$$
\varphi(t) \equiv \varphi_{r, i}(t):=\frac{\sin (t / 2)}{(\pi-t)^{2 r+2-i}}
$$

возрастает на $(0, \pi)$.

Стандартными методами проверяется, что функция

$$
\psi(t) \equiv \psi_{r, i}(t):=\frac{\cos (t / 2)}{(\pi-t)^{2 r+2-i}}
$$

также возрастает на $(0, \pi)$.

Отсюда и из (21) следует, что

$$
\begin{gathered}
\left|l_{i, j}\right|<\left|l_{i, j+1}\right|, \quad j=1, \ldots, n-1, \\
\operatorname{sign} l_{i, j}=-\operatorname{sign} l_{i, j+1}, \quad j=1, \ldots, n-1 .
\end{gathered}
$$

Положим

$$
\lambda_{i, j} \equiv \lambda_{i, j, n}:=\sum_{k=1}^{j} l_{i, k}, \quad j=1, \ldots, n .
$$

Применим преобразование Абеля к внутренней сумме в правой части (19):

$$
\begin{aligned}
\sum_{j=n-s+1}^{n} F\left(x_{j, n}\right) l_{i, j}= & F\left(x_{n, n}\right) \lambda_{i, n}+\sum_{j=n-s+1}^{n-1}\left(F\left(x_{j, n}\right)-F\left(x_{j+1, n}\right)\right) \lambda_{i, j} \\
& -F\left(x_{n-s+1, n}\right) \lambda_{i, n-s} .
\end{aligned}
$$


В силу $(22),(23)$ и (20) имеем

$$
\left|\lambda_{i, j}\right| \leqslant\left|l_{i, j}\right| \leqslant \frac{1}{\left(\pi-x_{j, n}\right)^{2 r+2-i}} .
$$

В частности,

$$
\left|\lambda_{i, n}\right| \leqslant\left|l_{i, n}\right| \leqslant \frac{1}{\left(\pi-\frac{2 n \pi}{2 n+1}\right)^{2 r+2-i}}=O\left(n^{2 r+2-i}\right), \quad n \rightarrow \infty .
$$

Отсюда с учетом того, что $F$ непрерьвна в точке $\pi$ и $F(\pi)=0$, следует, что

$$
F\left(x_{n, n}\right) \lambda_{i, n}=o\left(n^{2 r+2-i}\right) .
$$

Далее, в силу (25) и (22)

$$
\left|\lambda_{i, n-s}\right| \leqslant\left|l_{i, n-s}\right|<\left|l_{i, n}\right|=O\left(n^{2 r+2-i}\right), \quad n \rightarrow \infty,
$$

и в силу выбора $s$

$$
\begin{gathered}
\left|F\left(x_{n-s+1, n}\right) \lambda_{i, n-s}\right| \leqslant \varepsilon O\left(n^{2 r+2-i}\right), \quad n \rightarrow \infty \\
\left|\sum_{j=n-s+1}^{n-1}\left(F\left(x_{j, n}\right)-F\left(x_{j+1, n}\right)\right) \lambda_{i, j}\right| \leqslant V_{H}(F ; \pi-\delta, \pi) \max _{j=n-s+1, \ldots, n-1}\left|(n-j+1) \lambda_{i, j}\right| .
\end{gathered}
$$

Из (25) получаем

$$
\left|(n-j+1) \lambda_{i, j}\right| \leqslant \frac{n-j+1}{(\pi-2 j \pi /(2 n+1))^{2 r+2-i}} \leqslant(2 n+1)^{2 r+2-i} .
$$

Отсюда и из $(28)$ в силу выбора $\delta$ находим

$$
\left|\sum_{j=n-s+1}^{n-1}\left(F\left(x_{j, n}\right)-F\left(x_{j+1, n}\right)\right) \lambda_{i, j}\right| \leqslant \varepsilon(2 n+1)^{2 r+2-i} .
$$

Учитьвая $(24),(26),(27)$ и последнее неравенство, будем иметь

$$
\sum_{j=n-s+1}^{n} F\left(x_{j, n}\right) l_{i, j}=o\left(n^{2 r+2-i}\right), \quad n \rightarrow \infty
$$

откуда и из (19) следует, что

$$
\sum_{j=n-s+1}^{n} F\left(x_{j, n}\right) d_{j}=o\left(n^{2 r+2}\right), \quad n \rightarrow \infty,
$$

и, значит, $B_{n}=o\left(n^{2 r+1}\right)$. Аналогично доказьвается, что и $E_{n}=o\left(n^{2 r+1}\right), n \rightarrow \infty$. Отсюда из (13), (12) и (9) следует (6), т.е. утверждение а) теоремы 1 доказано.

Что касается утверждения б), то оно следует из того, что для всякой нечетной $2 \pi$-периодической функции $f$ справедливо равенство $L_{n}^{(2 r)}(f ; \pi)=0$ независимо от того, имеет ли $f$ скачок в точке $\pi$ или нет.

Отметим, что утверждение а) теоремы 1 настоящей работы было анонсировано без доказательства в [14]. 


\section{СПИСОК ЦИТИРОВАННОЙ ЛИТЕРАТУРЫ}

[1] Голубов Б. И. Определение скачка функции ограниченной $p$-вариации по ее ряду Фурье // Матем. заметки. 1972. Т. 12. №1. С. 19-28.

[2] Wiener N. The quadratic variation of a function and its Fourier coefficients // J. Math. Phys. 1924. V. 3. P. 72-94.

[3] Fejer L. Über die Bestimmung des Sprunges der Funktion aus Fourierreihe // J. Reine Angew. Math. 1912. V. 142. № 1. P. 165-188.

[4] Czillag P. Über die Fourierkonstanten einer Funktion von Beschränkter Schwankung // Mat. Phys. Lapok. 1918. V. 27. P. 301-308.

[5] Зигмунд А. Тригонометрические ряды. Т. І. М.: Мир, 1965.

[6] Avdispahić $M$. On the determination of the jump of a function by its Fourier series // Acta Math. Hung. 1986. V. 48. № 3-4. P. 267-271.

[7] Kvernadze G. Determination of the jumps of a bounded function by its Fourier series // J. Approx. Theory. 1998. V. 92. № 2. P. 167-190.

[8] Waterman D. On convergence of Fourier series of functions of generalized bounded variation // Studia Math. 1972. V. 44. № 2. P. 107-117.

[9] Кельзон А. А. Определение скачка функции ограниченной $p$-вариации с помощью тригонометрического интерполирования. Казань, 1977. Деп. в ВИНИТИ № 2434-78 Деп.

[10] Натансон И. П. Конструктивная теория функций. М.-Л.: ГИТТЛ, 1949.

[11] Градштейн И. С., Рыжик И. М. Таблицы интегралов, сумм, рядов и произведений. М.: Наука, 1971.

[12] Waterman D. On the summability of Fourier series of functions of $\Lambda$-bounded variation // Studia Math. 1976. V. 55. №1. P. 87-95.

[13] Фихтенгольц Г. М. Курс дифференциального и интегрального исчисления. Т. ІІ. СПб.: Лань, 1997.

[14] Кельзон А. А. Определение скачка функции обобщенной ограниченной вариации с помощью тригонометрического интерполирования // Гос. морская академия им. адм. С. О. Макарова. Научно-техн. конф. проф.-преп. состава и научных сотрудников. Тезисы докладов: СПб, 2001. C. $174-175$. 